\title{
An Algorithm for the Evaluation of the Dynamics of Blood Flow in the Carotid Arteries and the Air Flow in the Trachea of the Resuscitated Patient
}

\author{
Artem Sh. Bureev, Dmitry S. Zhdanov, Ekaterina Yu. Kiseleva, Mikhail S. Kutsov \\ National Research Tomsk State University \\ Tomsk, Russia \\ eka.kiselyova@gmail.com
}

\begin{abstract}
The article is to study evaluation algorithms of the dynamics of blood flow and air flow recorded in the bifurcation area of the carotid artery and trachea, underlying in the operation of the device for the control of cardiopulmonary resuscitation procedure of a human.
\end{abstract}

Keywords - cardiopulmonary resuscitation, evaluation of blood flow dynamics, assessment of breathing dynamics, carotid bifurcation, trachea, analysis acoustic signal.

\section{INTRODUCTION}

Sudden cardiac death is the leading cause of death in the world. Thus, in the US, the number of sudden cardiac death is 1 case per 1000 people per year, while in Russia - up to 2 cases per 1000 people. Emergency assistance for people with cardiac failure - sudden cardiac death - remains an urgent problem not only in emergency medicine, but also in therapy and cardiology in particular $[1,2,3]$.

According to the statistical data, up to $90 \%$ of the patients who faced clinical death could survive, if they received adequate medical care within the first 5 minutes after cardiac arrest. Unfortunately, even at large medical institutions, physicians often cannot perform the cardiopulmonary resuscitation $(\mathrm{CPR})$ procedure in compliance with recommendations.

Mortality reducing is an important task of providing emergency medical care. According to [4], most people do not have sufficient skills in cardiopulmonary resuscitation. Therefore, devices allowing a person, performing the procedure of cardiopulmonary resuscitation (hereinafter - CPR), to obtain feedback about the correctness of the procedure, are in high demand.

Currently in the market there are no mobile devices for non-stop real-time evaluation of the adequacy of resuscitation and for the control of the patient's state during and after the procedure.

The design team of the Professional Equipment Engineering Research Laboratory of National Research Tomsk State University are working on the development of an experimental sample of the device for the control of CPR (hereinafter - the device [5]). The device is aimed at analysis of acoustic blood flow signals and air flow recorded in bifurcation area of the carotid artery and trachea for evaluating the correctness and adjustment of the actions of the personnel conducting CPR.

The operating mode of the device is determined by availability of the vital functions of the patient:

- Monitoring mode: cyclical check of vital functions of the patient;

- Resuscitation mode: evaluating the correctness and adjustment of the actions of the resuscitator.

Implementation of the above-described principle requires complex software algorithms for assessment of the dynamics of blood flow in the carotid arteries and air flow in the throat of the resuscitated patient by means of analyzing acoustic signals. The algorithms allow detecting the presence of vital functions in case of a positive effect of the resuscitation. Operation of the device in Monitoring mode allows a resuscitator to determine the time of a subsequent cardiac arrest urgently. The vital functions of a patient in Monitoring mode are determined during the assessment of the blood flow in the bifurcation area of the carotid artery and air flow in the patient's trachea.

\section{ASSESSMENT ALGORITHM OF THE DYNAMICS OF BLOOD FLOW IN THE CAROTID ARTERIES}

Currently only Doppler-based ultrasonic angiography is used for determination of the blood flow dynamics. However, the use of the Doppler Effect For determination of the true blood flow speed (dynamics) in the carotid arteries will complicate the design of the sensor due to the required angle between the ultrasound transducer and the patient's neck.

In addition, application of an ultrasonic transmitter and receiver is not possible without immersion liquid necessary to avoid ultrasonic attenuation which is unacceptable during the resuscitation as it may require extra time for fixing the sensors on the patient's neck. Thus, the design team found a piezoelectric transducer the most suitable for receiving acoustic signals from the patient's neck.

As the device evaluates the acoustic blood flow signal, its dynamics can be evaluated with a blood beat number in the area of division of the carotid arteries for a certain period of time. 
In Monitoring mode evaluation algorithm for noise recorded in the bifurcation of the carotid artery is applied. Blood flow dynamics can be taken as a binary function that can be valued as:

- 0 if there is no heart;

- 1 if there is a heartbeat.

Figure 1 shows a graphic of the function describing the dynamics of the blood flow in the carotids.

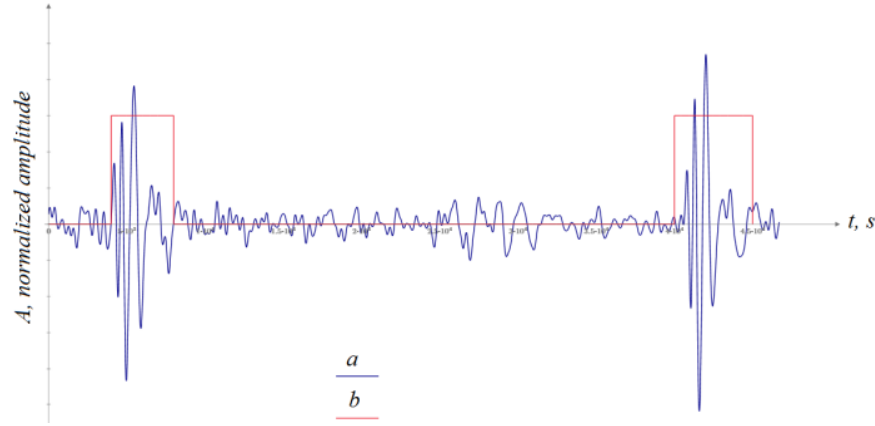

Fig. 1. Function describing the dynamics of the blood flow in the carotids. (a - acoustic signal recorded in the bifurcation area of the carotid arteries; $b$ - a binary function showing the blood flow dynamics.)

After a positive effect of the resuscitation a patient may have bradycardia (heart rate about $25-60$ beats per minute).

Thus the minimum number of blood flow beats, registered by acoustic sensors in the bifurcation area of the carotid arteries, will be 12 .

Within 30 seconds, in Monitoring mode the number of blood beats in the area of carotid bifurcation is counted.

Figure 2 shows the basic block diagram of the algorithm for estimating the dynamics of air flow in the trachea.

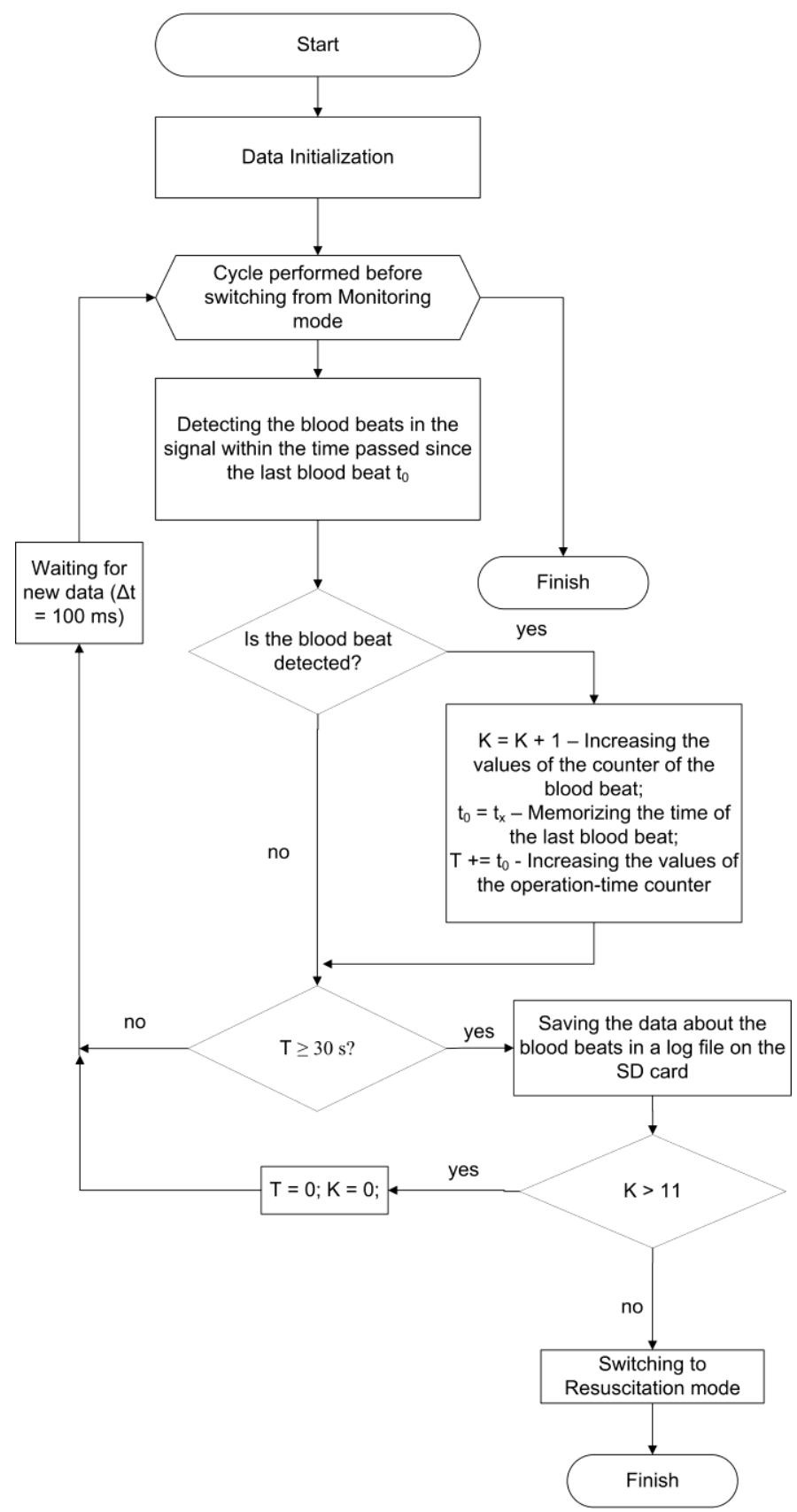

Fig. 2. The basic block diagram of the algorithm for estimating the dynamics of air flow in the trachea.

The algorithm of blood flow dynamics evaluation in the area of carotid bifurcation is performed continuously in the Monitoring mode and starts immediately when the device is in this mode. The sequence of cyclical actions:

1) to prepare variables, reset to 0 their values;

2) to perform a sequence of actions when device is turned on and is in Monitoring mode:

a) to determine the presence of blood beats in the area of carotid bifurcation in the signal section from t0 to tx, where $\mathrm{t} 0$ time of the end of the last blood beat found, tx - the current time of the end of the signal;

b) If the blood beat is not detected, go to step d; 
c) if blood beat is detected, memorize the new time of the end of the last beat found $\mathrm{t} 0, \mathrm{~F}$, increase blood counter;

d) If the operation time $\mathrm{T}$ of the cycle is less than 30 seconds, go to step 3;

e) If the operation time $\mathrm{T}$ of the cycle is more or equal to 30 seconds, save the current data about the blood flow in a log file on the SD card;

f) if the number of blood beats in the area of carotid bifurcation $\mathrm{K}$ is more than zero, reset variables of the cycle time $\mathrm{T}$ to 0 and the number of blood beats $\mathrm{K}$, go to step 3;

$\mathrm{g}$ ) if the number of blood beats $\mathrm{K}$ equals zero, put the device in Resuscitation mode and complete the operation work cycle;

3) wait for the new acoustic data of the blood flow for a while and continue performing from step 2.

The basic block diagram of the algorithm for estimating the dynamics of air flow in the trachea is shown in Figure 2.

Thus, as soon as the device detects a patient's heart rate lower than 24 beats per second, a sound signal alarms a resuscitator about the possible need to start the resuscitation procedure.

\section{ALGORITHM FOR ESTIMATING THE DYNAMICS OF AIR FLOW IN THE TRACHEA OF THE RESUSCITATED PATIENT}

At the moment for the registration of the breathing characteristics, including air flow rate, spirography and plethysmography are generally used.

Spirography is a graphic registration of changes in lung volume during respiration. Plethysmography allows measuring volume of thoracic gas, calculating airway resistance of the respiratory tract and estimating the shape of the resistance curve $[6,7]$.

However, the these methods can not be used in the device for controlling the CPR procedures as they need additional devices for data processing. It certainly leads to an increase of time of preparation, which is unacceptable in the critical situation of saving of a human life.

The most suitable method for assessing the dynamics of air flow in the trachea in terms of reliability and technical feasibility is the method of analysis of the acoustic signals received from the surface between the presternum and shieldlike cartilage on the trachea of the resuscitated patient.

It can be assumed that the air flow dynamics in the trachea is presence or absence of air flow in the trachea for 30 seconds.

According to the functioning algorithm of the Device, evaluation of air flow speed [5] in the trachea of the resuscitated patient is performed in Monitoring mode in the survey of acoustic sensors every 30 seconds.

Thus, the dynamics of air flow in the trachea is considered in terms of number breaths within 30 seconds.

A healthy adult in a state of physiological dormancy averagely performs 16 to 20 breaths per minute. In the dream, breathing slows down to $12-14$ breaths per minute, and during physical activity, emotional excitement or after eating it naturally quickens [8].
In case of a positive response of the patient to the CPR respiratory rate acceleration (tachypnea) or respiratory rate deceleraton (bradypnoea) can occur.

In case of tachypnea respiratory rate reaches more than 20 breathing cycles per 1 minute for an adult. In case of bradypnea respiratory rate reaches $10-12$ or even fewer breathing cycles per 1 minute.

In case of bradypnea after the resuscitation, within 30 seconds (in the worst case scenario) only one respiratory cycle can be found.

Figure 3 shows a real acoustic respiration signal. Thus, the dynamics of air flow in the trachea can be taken as a binary function, which equals 1 in the presence of acoustic breath sounds and 0 in their absence. The signal of dynamics is shown in Figure 3 (curve - 1).

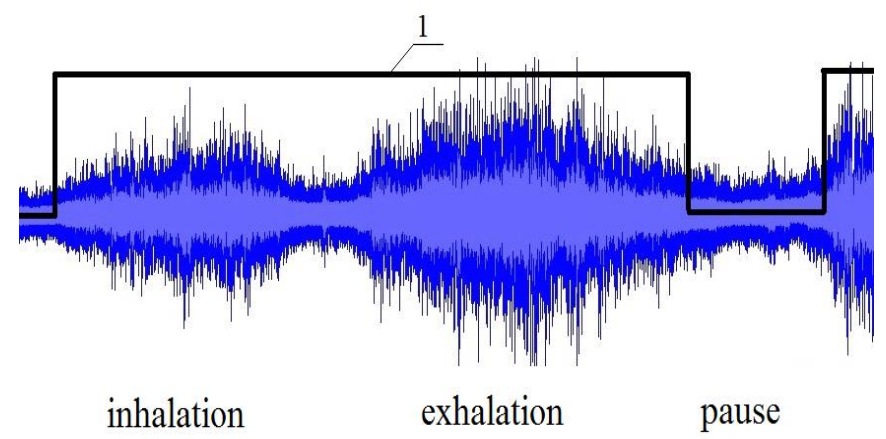

Fig. 3. The acoustic signal of respiratory noise $(1-$ The function of the dynamics of air flow in the trachea).

Thus, the algorithm of dynamics estimation of air flow in the trachea aims at calculation of the respiratory sounds within 30 seconds (Figure 7). If during those 30 seconds there are no respiratory noise signal found, the Device enters Resuscitation mode. In other cases, the Device operates in Monitoring mode.

The algorithm of dynamics estimation of air flow in the trachea (Figure 4) is applied during the entire time of the device operating in Monitoring mode and starts immediately when the device is in this mode. The sequence of cyclical actions:

1) to prepare variables, reset to 0 their values;

2) to perform a sequence of actions when device is turned on and is in Monitoring mode:

a) to determine the presence of respiratory movements in the area of the signal from $t 0$ to $t x$, where $t 0$ - time of the end of the last respiratory movement found, $t x$ - the current time of the end of the signal;

b) if the respiratory movement is not detected, go to step d);

c) if a respiratory movement is detected, memorize the new time of the end of the last respiratory movement found $t 0$, increase the operation-time counter, increase respiratory movements counter;

d) If the operation time $\mathrm{T}$ of the cycle is less than 30 seconds, go to step 3;

e) If the operation time $\mathrm{T}$ of the cycle is more or equal to 30 seconds, save the current data about the breathing in a log file on the SD card; 
f) if the number of respiratory movements $\mathrm{D}$ is more than 0 , reset to 0 cycle time variables $\mathrm{T}$ and number of breaths $\mathrm{D}$, go to step 3;

$\mathrm{g}$ ) if the number of respiratory movements $\mathrm{D}$ equals 0 , switch to a Resuscitation mode and complete the cycle operating;

3) wait for a while for the new acoustic respiration data and continue from step 2 .

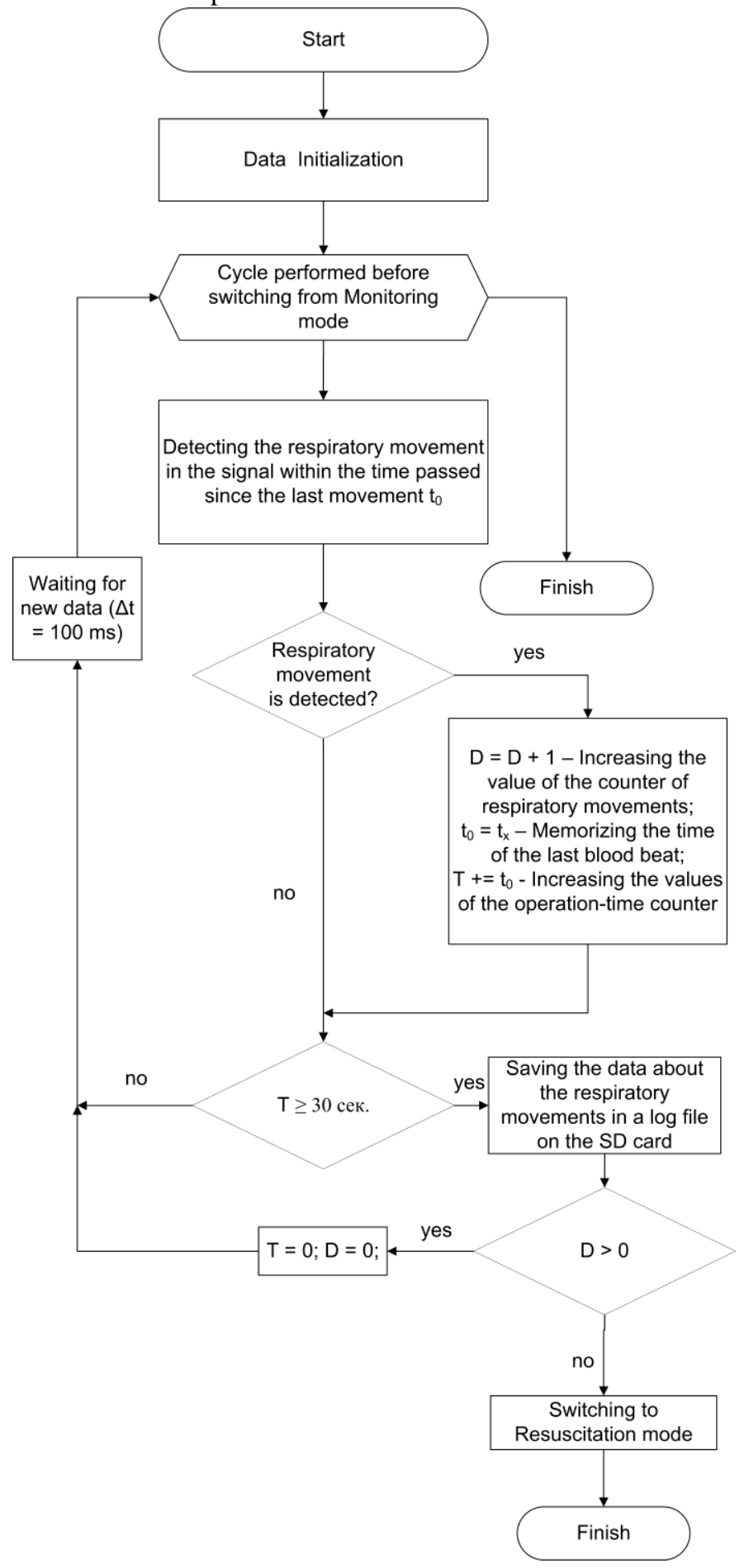

Fig. 4. The basic block diagram of the algorithm for estimating the dynamics of air flow in the trachea.

\section{CONCLUSION}

The result of the work presents a developed set of software algorithms for assessment of the dynamics of blood flow in the carotid arteries and air flow in the larynx of the resuscitated patient with the aid of acoustic signals analyzing.

The software runs as part of the device for the control of the CPR procedure of a human.

These algorithms developed operate simultaneously and allow resuscitators to detect the subsequent cardiac and respiratory arrest urgently. This will allow to start resuscitation procedure in time and improve the survival rate by reducing the reaction time of the resuscitator at the time of the subsequent clinical death.

\section{ACKNOWLEDGMENT}

The study was undertaken in the framework of the agreement № 14.578.21.0078 (unique identifier RFMEFI57814X0078) entitled "Device for control of a CPR procedure of a human", between the Ministry of Education and Science of the Russian Federation and the National Research Tomsk State University.

\section{REFERENCES}

[1] Bokerija L A, Bokerija O L, Kislicina O N (2010) Randomized clinical research aimed at prevention of sudden cardiac death: principles and results Annaly aritmologii 2 5-13 (in Russian)

[2] Kobylkin I S (2012) Review of quality control devices for performing of chest compressions during CPR Medicinskij alfavit. Neotlozhnaja medicina 4 58-62 (in Russian)

[3] Field J M, Hazinski M F, Sayre M R, Chameides L, Schexnayder S M, Hemphill R, Samson R A, Kattwinkel J, Berg R A, Bhanji F, Cave D M, Jauch E C, Kudenchuk P J, Neumar R W, Peberdy M A, Perlman J M, Sinz E, Travers A H, Berg M D, Billi J E, Eigel B, Hickey R W, Kleinman M E, Link M S, Morrison L J, O'Connor R E, Shuster M, Callaway C W, Cucchiara B, Ferguson J D, Rea T D, Vanden Hoek T L (2010) Part 1: executive summary: 2010 American Heart Association Guidelines for Cardiopulmonary Resuscitation and Emergency Cardiovascular Care Circulation 122 (3) S640-S656

[4] B.S. Abella, J.P. Alvarado, H. Myklebust, D.P. Edelson, A. Barry, N. O'Hearn, T.L. Vanden Hoek, L. B. Becker. Quality of Cardiopulmonary Resuscitation During InHospital Cardiac Arrest. JAMA. 2005; 293(3):305-310.

[5] A. Sh. Bureev, D. S. Zhdanov, E. Yu. Kiseleva, M. S. Kutsov, and A. Yu. Trifonov, "A method of automatic control procedures cardiopulmonary resuscitation" New Operational Technologies (NewOT'2015) AIP Conf. Proc. 1688, 040005-1-040005-5.

[6] E.N. Kalmanova The study of the respiratory function in patients with lung diseases // Atmosphere. Pulmonology and Allergology, 2002. №2. - pp. 14-17.

[7] Guidelines on physiology. Breathing physiology / Ed. A.L. Chernigov: Nauka, 1973. - 352 p.

[8] Propedeutics of internal diseases / Ed. V.H. Vasilenko. 3rd ed. - Moscow : Medicine, 1989. - 51 
